\title{
Short-distance part of the interaction of $D$ mesons and nucleons
}

\author{
G. Krein* \\ Instituto de Física Teórica, Universidade Estadual Paulista \\ Rua Pamplona, 145 - 01405-900 São Paulo, SP - Brazil \\ E-mail: gkreineift.unesp.br \\ V. E. Vizcarra \\ Instituto de Física Teórica, Universidade Estadual Paulista \\ Rua Pamplona, 145 - 01405-900 São Paulo, SP - Brazil \\ E-mail: indhy@ift.unesp.br
}

\begin{abstract}
The short-distance part of the low energy interaction of D-mesons and nucleons is investigated in the context of a quark model. The quark model is based on Coulomb gauge QCD. The model contains a confining Coulomb potential and a transverse hyperfine interaction consistent with a finite gluon propagator in the infrared. The basic mechanism for the short-distance interaction between the D-mesons and nucleons is quark interchange. Using Resonating Group Method techniques an effective potential for the interaction between nucleons and D mesons can be obtained and used in a Lippmann-Schwinger equation to obtain differential cross-sections and phase shifts.
\end{abstract}

8th Conference Quark Confinement and the Hadron Spectrum September 1-6 2008

Mainz, Germany

${ }^{*}$ Speaker. 


\section{Introduction}

Heavy-light mesons, like the $D$-mesons, are interesting bound states of quantum chromodynamics (QCD). They are interesting because to a good approximation they can be described as one-body bound states, since the charm $c$ quark is much heavier than the light $u$ and $d$ quarks, a feature that simplifies tremendously their mathematical treatment. They are also interesting for studying chiral symmetry in medium. The light quarks are sensitive to temperature and baryon density and studies of the propagation of $D$ mesons in hadronic matter [1] can provide valuable information on possible changes of chiral symmetry in medium. However, as emphasized in recent publications [2,3], before one can hope to extract reliable information on chiral changes inmedium, it is necessary to understand the interaction of $D$ mesons with nucleons in free space. In these publications, the free-space interaction of $D$ mesons with nucleons was studied in the context of a hybrid model, where the long-distance part of the interaction was described by meson-baryon exchange, and the short-distance part was described by quark-gluon-interchange in the context of a nonrelativistic quark model.

Different nonrelativistic quark models share the common feature that baryons and mesons are bound states of constituent quarks and antiquarks with masses of the order of $300 \mathrm{MeV}$. Also, the quarks and antiquarks are confined by a prescribed potential, and residual interactions derived from perturbative one-gluon exchange (OGE) provide a means to describe spin-dependent mass splittings. Given the microscopic interactions and the bound state wave functions, low-energy effective hadronic interactions can be derived within such a model using a variety of methods, the most well known being the resonating group method (RGM) - for a review and a list of references on the RGM and other methods, see e.g. Ref. [4]. Now, although such a framework has proved to be very useful in many instances, it is clearly very limited for studying effects related to in-medium changes of hadron properties. For that one needs a model that is closer to QCD, but still simple enough so that one can make calculations without resorting to extensive numerical simulations. A model that provides such a workable framework is based on the QCD Hamiltonian in Coulomb gauge [5]. In the present communication we use such a model to construct a calculational scheme to obtain effective hadron-hadron interactions in close analogy to the traditional approaches [4] based on the nonrelativistic quark model. The model is based on a field theoretic Hamiltonian that confines color and realizes dynamical chiral symmetry breaking. The model allows to construct an approximation scheme for defining hadronic bound-state wave functions and effective hadronhadron potentials can be derived using the RGM. The effective hadron-hadron potentials can be used in a Lippmann-Schwinger equation to obtain cross-sections and phase-shifts.

We use the formalism to obtain a short-distance potential for $\bar{D}$-nucleon system and calculate cross-sections and phase shifts at low energies - close to threshold. A recent publication [3] investigated the possibility to extract information about the $\bar{D} N$ interaction from the $\bar{p} d \rightarrow D^{0} D^{-} p$ reaction using for the short distance part of the $\bar{D} N$ interaction an effective interaction based on the OGE. There are proposals for experiments of this kind by the PANDA collaboration at the FAIR facility at the GSI laboratory in Germany. Our calculation serves the purposes of comparing results with the traditional approach based on OGE, and also to provide a framework to extend the present model to study in-medium hadron-hadron interactions. 


\section{The model}

The microscopic Hamiltonian of the model is given in terms of quark $\Psi(\mathbf{x})$ and transversegluon $\mathbf{A}^{a}(\mathbf{x}), a=1, \cdots, 8$, field operators [6,7]. Since the present work focuses on quark degrees of freedom, the relevant part of the Hamiltonian involves only $\Psi(\mathbf{x})$ and it can be written as

$$
\begin{aligned}
H & =\int d \mathbf{x} \Psi^{\dagger}(\mathbf{x})\left(-i \alpha \cdot \nabla+\beta m_{0}\right) \Psi(\mathbf{x})-\frac{1}{2} \int d \mathbf{x} d \mathbf{y} \rho^{a}(\mathbf{x}) V_{C}(|\mathbf{x}-\mathbf{y}|) \rho^{a}(\mathbf{y}) \\
& +\frac{1}{2} \int d \mathbf{x} d \mathbf{y} J_{i}^{a}(\mathbf{x})\left(\delta_{i j}-\frac{\nabla_{i} \nabla_{j}}{\nabla^{2}}\right) V_{T}(|\mathbf{x}-\mathbf{y}|) J_{j}^{a}(\mathbf{y})
\end{aligned}
$$

where $m_{0}$ is the current quark matrix (in the limit of exact chiral symmetry, $m_{0}=0$ ), and $\rho^{a}(\mathbf{x})=$ $\Psi^{\dagger}(\mathbf{x}) T^{a} \Psi(x)$ and $J_{i}^{a}(\mathbf{x})=\Psi^{\dagger}(\mathbf{x}) T^{a} \alpha_{i} \Psi(\mathbf{x})$ are the color charge and current densities, with $T^{a}=$ $\lambda^{a} / 2$, where $\lambda^{a}$ are the $S U(3)$ Gell-Mann matrices. Once $V_{C}$ and $V_{T}$ are given the Hamiltonian is completely specified. Next, one expands the field operators as

$$
\Psi(\mathbf{x})=\int \frac{d \mathbf{k}}{(2 \pi)^{3 / 2}} \sum_{s= \pm 1 / 2}\left[u_{s}(\mathbf{k}) q_{s}(\mathbf{k})+v_{s}(\mathbf{k}) \bar{q}_{s}^{\dagger}(-\mathbf{k})\right] e^{i \mathbf{k} \cdot \mathbf{x}}
$$

where $q_{s}^{\dagger}(\mathbf{k}), \bar{q}_{s}^{\dagger}(-\mathbf{k}), q_{s}(\mathbf{k})$ e $\bar{q}_{s}(-\mathbf{k})$ represent creation and annihilation operators of constituent quarks, and

$$
u_{s}(\mathbf{k})=\sqrt{\frac{E_{k}+M_{k}}{2 E_{k}}}\left(\begin{array}{c}
1 \\
\frac{\sigma \cdot \mathbf{k}}{E_{k}+M_{k}}
\end{array}\right) \chi_{s}, \quad v_{s}(\mathbf{k})=\sqrt{\frac{E_{k}+M_{k}}{2 E_{k}}}\left(\begin{array}{c}
-\frac{\sigma \cdot \mathbf{k}}{E_{k}+M_{k}} \\
1
\end{array}\right) \chi_{s}^{c}
$$

with $E_{k}=\left(k^{2}+M_{k}^{2}\right)^{1 / 2}, \chi_{s}^{c}=-i \sigma^{2} \chi_{s}^{*}$, and $\chi_{s}$ is a Pauli spinor. The momentum dependent function $M_{k}$ is the constituent quark mass function. The mass function is solution of a nonlinear integral equation - the quark gap equation - which can be obtained by using Wick contraction techniques. The contracted Hamiltonian can be written as $H=H_{0}+H_{2}+H_{4}$, where $H_{0}$ is a c-number, the vacuum energy, and $\mathrm{H}_{2}$ and $\mathrm{H}_{4}$ are normal ordered terms with two and four field operators $\Psi$. The normal ordering is with respect to the constituent quark creation and annihilation operators of Eq. (2.2). $H_{2}$ contains self-energy contributions and is infrared divergent for $V_{C}$ confining. $H_{4}$ also contains an infrared divergence for $V_{C}$ confining. These infrared divergences cancel in color singlet bound states [8].

The Wick contracted Hamiltonian can be used to obtain hadron bound states with the variational principle [8]. One can simplify considerably the approach without sacrificing the physical content of the model using a low momentum expansion for the mass function $M_{k}$ as

$$
M_{k}=M-M_{1} k-M_{2} k^{2}
$$

where $M=M(k=0)$. Retaining terms up to $\mathscr{O}\left(k^{2} / M^{2}\right)$ in the expansion of the spinors $u_{s}(\mathbf{k})$ and $v_{s}(\mathbf{k})$, one obtains a Fermi-Breit type of Hamiltonian. For the nucleon and D-mesons ground states the most important components of these interactions can be written in momentum space as

$$
V(\mathbf{q})=V_{C}(\mathbf{q})+\frac{2}{3} \frac{q^{2}}{M_{1} M_{2}} \mathbf{S}_{1} \cdot \mathbf{S}_{2} V_{T}(\mathbf{q})
$$


where $V_{C}(\mathbf{q})$ and $V_{T}(\mathbf{q})$ are the Fourier transforms of $V_{C}(|\mathbf{x}|)$ and $V_{T}(|\mathbf{x}|)$. We take Gaussian forms for the nucleon and D-meson wave functions - see Eqs. (16)-(18) of Ref. [2] - where the width parameters $\alpha$ and $\beta$ are taken as variational parameters. Minimizing the expectations values of the Hamiltonian with these wave functions gives nonlinear equations for the parameters $\alpha$ for the nucleon and $\beta$ for the D-mesons (here we use $M_{u}=M_{d}$ )

$$
\begin{gathered}
\alpha^{4}=\frac{M_{u}}{2} \int \frac{d \mathbf{q}}{(2 \pi)^{3}} q^{2}\left[V_{C}(q)+\frac{2}{3} \frac{q^{2}}{M_{u}^{2}}\left(-\frac{1}{4}\right) V_{T}(q)\right] e^{-q^{2} / 2 \alpha^{2}} \\
\beta^{4}=\frac{2}{9}\left(\frac{M_{u} M_{c}}{M_{u}+M_{c}}\right) \int \frac{d \mathbf{q}}{(2 \pi)^{3}} q^{2}\left[V_{C}(q)+\frac{2}{3} \frac{q^{2}}{M_{u} M_{c}}\left(-\frac{3}{4}\right) V_{T}(q)\right] e^{-q^{2} / 4 \beta^{2}}
\end{gathered}
$$

The use of Gaussian wave functions is useful for getting closed forms for the effective hadronhadron interactions as derived with the RGM [4]. Their explicit expressions are the same as for the case of OGE given in Ref. [2], but with $v_{C}(q)$ and $v_{T}(q)$ of this reference replaced by our $V_{C}(q)$ and $V_{T}(q)$ of Eq. (2.5) above. Of course, the big difference here as compared to Ref. [2] is that the constituent quark masses $M_{u}$ and $M_{c}$, the width parameters $\alpha$ and $\beta$, and the interactions $V_{C}$ and $V_{T}$ are all derived from a single microscopic quark-gluon Hamiltonian.

\section{Numerical Results}

For an explicit application we need to specify $V_{C}$ and $V_{T}$. We use two forms for $V_{C}$. One, which we call model SS, is derived in Ref. [6] using a quasi-particle variational approach for the gluonic vacuum functional. The other form, which we call model Latt, is a fit to recent lattice simulations in Coulomb gauge QCD [9]. Specifically, we use the parametrization given in Eq. (92) of Ref. [6], its long distance part gives confinement in the form of an almost linearly rising potential and its short distance part that is asymptotically free, both match at some scale $m_{g}=600 \mathrm{MeV}$ - see also Ref. [7]. The long distance part of the lattice result is parameterized as [9]

$$
V_{C}(q)=\frac{8 \pi \sigma}{q^{4}}+\frac{4 \pi C}{q^{2}}
$$

with $\sigma=(552 \mathrm{MeV})^{2}$ and $C=6$. For the short distance part, we take for simplicity the same form as of the model SS and enforce that both parts match at $m_{g}=600 \mathrm{MeV}$. For the transverse part, we use the simple form

$$
V_{T}(q)=-\frac{4 \pi \alpha_{T}}{\left(q^{2}+\mu^{2}\right) \ln ^{1.42}\left(\tau+q^{2} / m_{g}^{2}\right)}
$$

The exponent of the log is inspired by the short distance part of $V_{C}$ and $\mu \neq 0$ is to conform with recent lattice results that show that the transverse gluon propagator is finite at $q^{2}=0$. The parameters $\alpha_{T}, \mu$ and $\tau$ are chosen to get a reasonable value for the quark condensate and a constituent quarks mass of $M_{u}=300 \mathrm{MeV}$. We use $\mu=m_{g} / 2, \tau=1.05$ and $\alpha_{T}=0.7$.

We have solved the quark gap equation and obtained $M_{u}$ and $M_{c}$. Given the constituent quark masses, we obtained the variational parameters $\alpha$ and $\beta$. While both $V_{C}$ and $V_{T}$ are equally important for the numerical values of $M_{u}$ and $M_{c}$, it is $V_{C}$ that essentially determines the values of $\alpha$ and $\beta$. Given the quark masses and the size parameters of the wave functions, we obtained the 

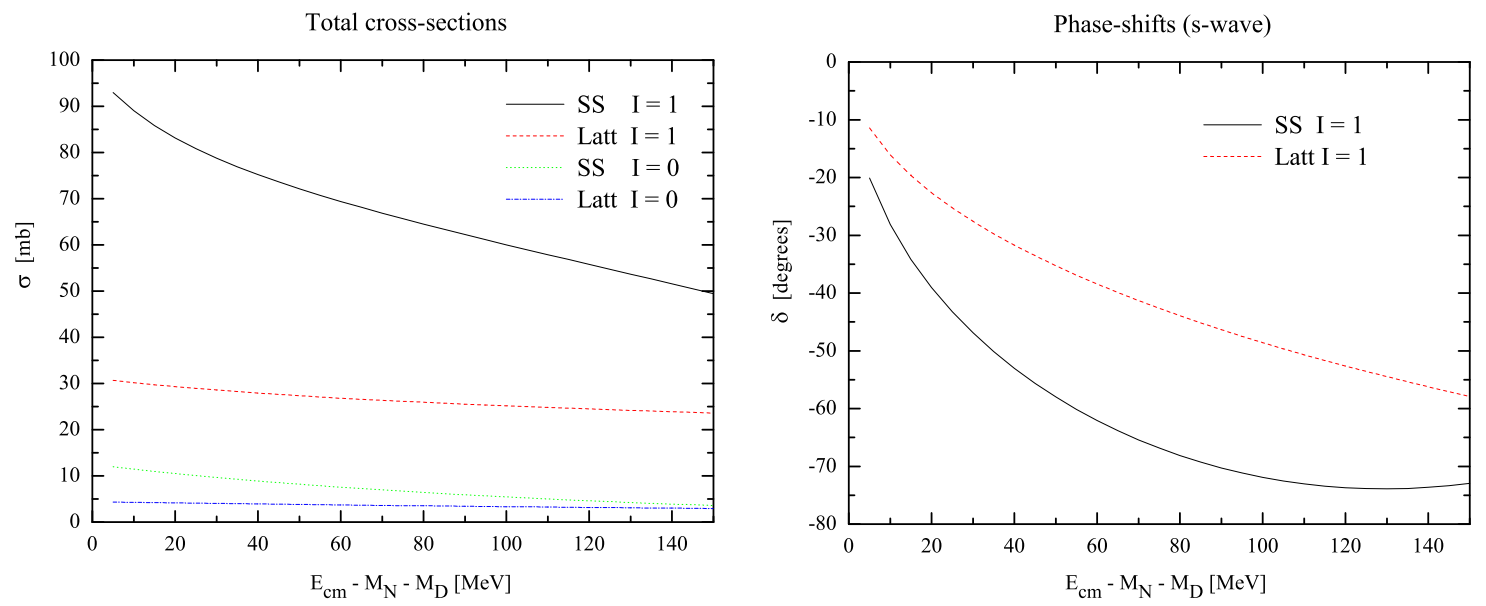

Figure 1: Total cross-sections for $I=1$ and $I=0$ (left panel) for the SS and lattice models, and $s$-wave phase-shifts for the SS model (right panel).

numerical values for the effective potentials for the nucleon- $\bar{D}^{0}$ and nucleon- $D^{-}$interactions and solved the Lippmann-Schwinger equation to calculate cross-sections and phase-shifts. In Fig. 1 we present a selected set of results. The SS model provides much larger values for the observables and, like for the case of the one-gluon exchange, the $I=1$ observables are much larger, because the spin-isospin factors for the Coulomb part are zero [2].

Concluding, in this communication we have sketched a calculational scheme to obtain effective hadron-hadron interactions from a microscopic quark-gluon Hamiltonian that confines quarks and gluons and realizes dynamical chiral symmetry breaking. We have applied the formalism to obtain the short-distance part of an effective nucleon $-\bar{D}^{0}$ and nucleon- $D^{-}$interaction.

Acknowledgements: Work partially financed by CNPq and FAPESP (Brazilian agencies).

\section{References}

[1] L. Tolos, J. Schaffner-Bielich and A. Mishra, Phys. Rev. C 70, 025203 (2004); T. Mizutani and A. Ramos, Phys. Rev. C 74 (2006) 065201.

[2] J. Haidenbauer, G. Krein, U. G. Meissner and A. Sibirtsev, Eur. Phys. J. A 33 (2007) 107.

[3] J. Haidenbauer, G. Krein, U. G. Meissner and A. Sibirtsev, Eur. Phys. J. A 37 (2008) 55 .

[4] D. Hadjimichef, G. Krein, S. Szpigel and J. S. da Veiga, Annals Phys. 268 (1998) 105.

[5] A. P. Szczepaniak and E. S. Swanson, Phys. Rev. Lett. 87 (2001) 072001.

[6] A. P. Szczepaniak and E. S. Swanson, Phys. Rev. D 65 (2002) 025012.

[7] D. Epple, H. Reinhardt, W. Schleifenbaum and A. P. Szczepaniak, Phys. Rev. D 77 (2008) 085007.

[8] P. J. A. Bicudo, G. Krein, J. E. F. Ribeiro and J. E. Villate, Phys. Rev. D 45 (1992) 1673.

[9] A. Voigt, E. M. Ilgenfritz, M. Muller-Preussker and A. Sternbeck, Phys. Rev. D 78 (2008) 014501. 